\title{
Ground-layer turbulence profiling using a lunar SHABAR
}

\author{
Anna Moore*a $^{\mathrm{a}}$, Eric Aristidi ${ }^{\mathrm{b}}$, Michael Ashley ${ }^{\mathrm{c}}$, Maurizio Busso ${ }^{\mathrm{d}}$, Maurizio Candidi ${ }^{\mathrm{e}}$, Jon Everett ${ }^{\mathrm{c}}$, \\ Suzanne Kenyon ${ }^{c}$, Jon Lawrence ${ }^{c}$, D, Luong-Van ${ }^{c}$, Andre Phillips ${ }^{c}$, Brice Le Roux ${ }^{f}$, Roberto \\ Ragazzoni ${ }^{\mathrm{f}}$, Piero Salinari ${ }^{\mathrm{f}}$, John Storey ${ }^{\mathrm{c}}$, Melinda Taylor ${ }^{\mathrm{c}}$, Gino Tosti ${ }^{\mathrm{d}}$, Tony Travouillon ${ }^{\mathrm{a}}$ \\ ${ }^{a}$ California Institute of Technology, Pasadena, CA 91125, USA \\ ${ }^{b}$ LUAN, Université de Nice, Parc Valrose, 06108 Nice Cedex 2, France \\ ${ }^{c}$ School of Physics, University of New South Wales, Sydney, NSW 2052, Australia \\ ${ }^{d}$ Dipartimento di Fisica, Università di Perugia, via A. Pascoli, 06123 Perugia, Italy \\ ${ }^{e}$ Istituto di Fisica dello Spazio, CNR, via Fosso Cavaliere 100, Roma, and Programma Nazionale di \\ Ricerche in Antartide, ENEA, Roma, Italy \\ ${ }^{f}$ Osservatorio Astrofisico di Arcetri, Largo Enrico Fermi, 5, Firenze 50125, Italy
}

\begin{abstract}
Profiling the ground layer turbulence for daytime seeing applications using an array of photodiodes has been documented in literature, in particular by Beckers who coined the term "SHABAR" for the instrument, short for Shadow Band Ranger. In this case the photodiodes measure the variation of solar intensity as a function of time and the correlation of scintillation between spatially separated scintillometers can be used to derive structure constant values for the lower $100 \mathrm{~m}$ or so. More recently SHABARs have been applied to night time atmospheric profiling using the moon as the extended source, such as the Pan-STARRS lunar SHABAR, a more challenging venture given the lower structure constant values and therefore higher sensitivity required. We present a summary of the lunar SHABAR currently operating at the Antarctic site of Dome C, one of the three Gattini site testing instruments for the Italian-led IRAIT project. The SHABAR was designed with low noise performance in mind and for low temperature operation. Ground layer profiling is of particular importance at the Dome $\mathrm{C}$ site during winter-time as it is known the majority of the integrated seeing measured at ground level is created in a turbulent layer very close to the ground.
\end{abstract}

\section{INTRODUCTION}

The application of an array of scintillometers for probing the nature of ground layer turbulence was first implemented by [1] based on earlier pioneering work by [2]. In the case of [1] the instrument was given the name SHABAR standing for Shadow Band Ranger, a term referring to the sometimes witnessed shadow patterns observed during a solar eclipse. The SHABAR was initially used to probe the turbulence profile of the ground layer at various sites chosen for location of a solar observatory, where a large percentage of daytime seeing is almost solely located in the bottom few hundred meters. The SHABAR, by observing an extended source, is an ideal instrument for such testing as the sun provides an ever present extended target.

The application of a SHABAR to night-time ground layer profiling was first attempted by [3] and later by [4] for the Pan-STARRS project. The reduced scintillation produced by the ground layer, the lower intensity of the target and the changing phase of the target make a lunar SHABAR a non-optimum solution for this particular application, however, the simplicity and expense of the instrument to a large extent compensates. The Pan-STARRS team performed, in particular, a thorough analysis of the effects of changing lunar phase and we wait with great anticipation publication of the on-sky results.

*email to: amoore@astro.caltech.edu

Ground-based and Airborne Instrumentation for Astronomy, edited by lan S. McLean, Masanori lye, Proc. of SPIE Vol. 6269, 62695U, (2006) · 0277-786X/06/\$15 · doi: 10.1117/12.672640 


\subsection{SHABAR basics}

The basic principle of the lunar SHABAR is shown schematically in Figure 1. By observing an extended source, in this case $0.5^{\circ}$ for full moon, intensity variations measured at one location on the ground due to variation in the horizontal refractive index of the atmosphere, or scintillation, are weighted towards low altitudes. In the lower $\sim 100 \mathrm{~m}$ the scale of atmospheric perturbation becomes equivalent to that of the cross-sectional area of the lunar cone with vertex angle $0.5^{\circ}$. For point sources such as stars the correlation function favours a much higher altitude of the range of kilometres, a simple geometrical effect as a point source has a much smaller subtended cone angle. By arranging multiple scintillometers in an array one can use the observed correlation in the intensity measurements to probe the minimum altitude of the turbulence creating the variation. Scintillometers spaced far apart probe the highest turbulent altitudes as only here do the corresponding cones intersect.

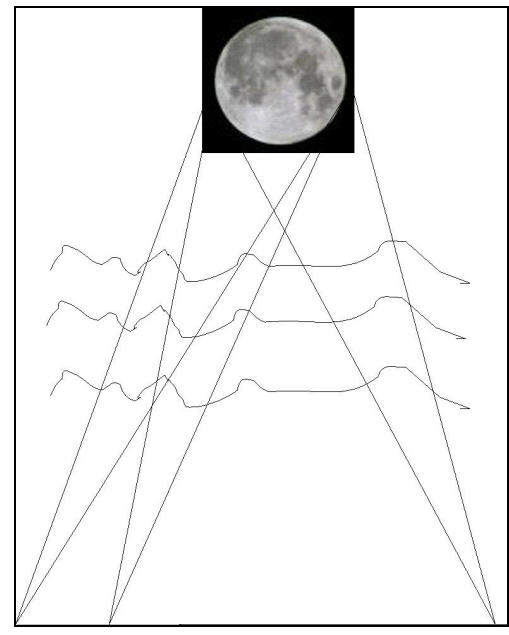

Figure 1: Schematic of the principle behind the lunar SHABAR. The measured scintillation from an extended source such as the moon is strongly sensitive to turbulence close to the ground where the size of the turbulent cells is equivalent

to the cross-sectional area of the cone. Observed coherence in the intensity fluctuations between spatially separated scintillometers ( 3 are shown above) can be used to probe the minimum height of this turbulence, where a maximum spacing gives information only on the highest layers where the corresponding cones intersect.

For the Dome C lunar SHABAR only the case of full moon is considered at this stage. For a moon at full phase the correlation function is fairly straightforward to calculate [1], [4]. For a moon at an intermediate phase the situation becomes more complicated as the subtended cones are no longer circularly symmetric. The correlation functions adopt an aziumthal dependence that still can be handled if the SHABAR is aligned to the long axis of the moon as described superbly in [4].

\subsection{Why Dome C?}

Since the release of spectacular seeing measurements at visible wavelengths $(550 \mathrm{~nm})$ taken at the Antarctic site of Dome $\mathrm{C}$ (location on the Antarctic plateau is shown in Figure 2 (right) during summertime at ground level [5] and during winter-time for high-altitude seeing [6] there have been several proposals for $2 \mathrm{~m}$ class optical telescopes that wish to exploit this unique location [7], [8], [9], as well as more ambitious projects [10], [11]. However, it is evident that a strong turbulent layer located very close to the ice is evident throughout the winter season. The maximum height of this layer has so far been limited to approximately $30-40 \mathrm{~m}$ or less from SODAR measurements [13], temperature profiles from balloon borne experiments and DIMM measurements [14]. The minimum height of this turbulent layer is an important parameter for optical telescopes and their associated structures that require access to the excellent seeing conditions existing only above.

\subsection{SHABAR location and logistics}

The lunar SHABAR is located close to the atmospheric laboratory approximately $1 \mathrm{~km}$ away from the French/Italian operated Concordia station, shown in Figure 2 (left). The SHABAR is mounted horizontally on a specially made wooden 
stand and is orientated to point towards the moon at maximum altitude during the winter season $\left(40^{\circ}\right.$ altitude, North). Figure 3 shows the general layout of some of the site testing instruments currently operating near the atmospheric laboratory, while a close-up of the lunar SHABAR is shown in Figure 4.
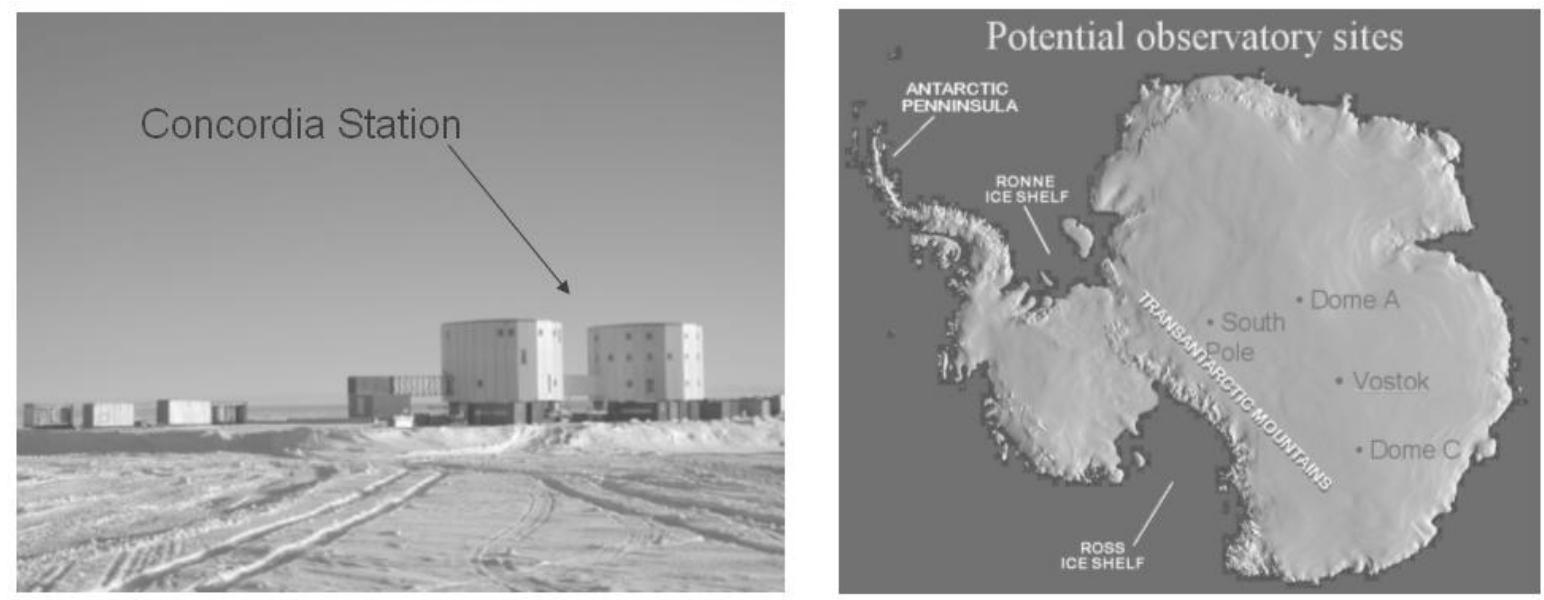

Figure 2: The French/Italian Concordia installation at the Dome $C$ site provides comfortable habitation and work environment for staff throughout the year (left); Relative locations of various high altitude sites including Dome C (right)

\section{SHABAR DESIGN}

\subsection{General}

The Dome C lunar SHABAR is transit in nature therefore contains no moving parts. This makes the instrument extremely cheap and robust, important qualities for an Antarctic location. The side effect is that the atmosphere probed as the moon traverses the sky varies with the net result that the distance to a turbulent layer, as measured by the SHABAR, must be multiplied by a factor proportional to the azimuthal angle of the moon. This can be accommodated so long as the real time clock of the supervisor computer is accurate (so far not an issue).

A similar effect occurs because the maximum elevation of the moon at the Dome $\mathrm{C}$ site is low compared to temperate locations, equivalent to $\sim 40^{\circ}$. A higher target altitude results in a smaller distance to the turbulent layer hence a higher scintillation index. However, with all things considered, the Dome C lunar SHABAR represents a cheap, easy to assemble solution for probing the height of the ground layer turbulence at discrete times at the Dome $\mathrm{C}$ site for winter 2006. These benefits far outweigh the complexity of providing a winterized telescope for dedicated tracking.

\subsection{Set-up}

The SHABAR consists of 7 photodiode units based on Seykora scintillometers [2]. The units are arranged in a nonredundant fashion in a linear array that is mounted horizontally, as shown in Figure 4. The array provides 21 sample baselines ranging from $\sim 30 \mathrm{~mm}$ to $1.5 \mathrm{~m}$. No filter is incorporated into the photodiode units. The SHABAR is located away from man-made turbulence (the heated lab, Concordia station) to avoid detecting anything other than turbulence created by the strong thermal gradient that exists during the winter combined with local wind.

\subsection{Electronic design}

Each photodiode unit is housed in a small steel container. Each unit is identical and composes (1) a $\sim 0.8 \mathrm{~cm}$ photodiode with no optical filter (2) a high pass filter (set to $\sim 3 \mathrm{~Hz}$ ) to filter slowly varying DC effects and (3) a high gain amplifier to increase photodiode voltage. The AC signal from each photodiode unit travels separately from the SHABAR location to the corresponding input lines of an analogue to digital card located in supervisor computer (15m away). Schottky diodes connected to each return line prevent damage to the input lines of the rather delicate analogue to digital card (from static build-up etc.). Finally, a low pass filter located inside the supervisor computer filters frequencies associated with the switch mode power supply. 
Electrolytic capacitors were avoided due to their decreased performance at low temperatures.

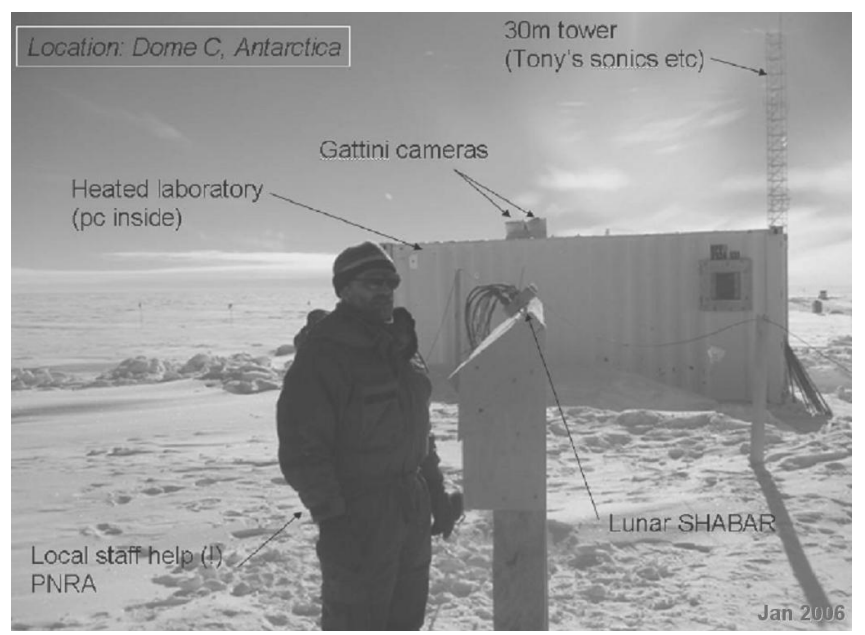

Figure 3: The lunar SHABAR is located close to the atmospheric laboratory at the Dome $\mathrm{C}$ site as shown above. The laboratory is a heated environment, a requirement for the control computer. In the foreground the transit lunar SHABAR points northwards at an elevation of $\sim 40^{\circ}$.

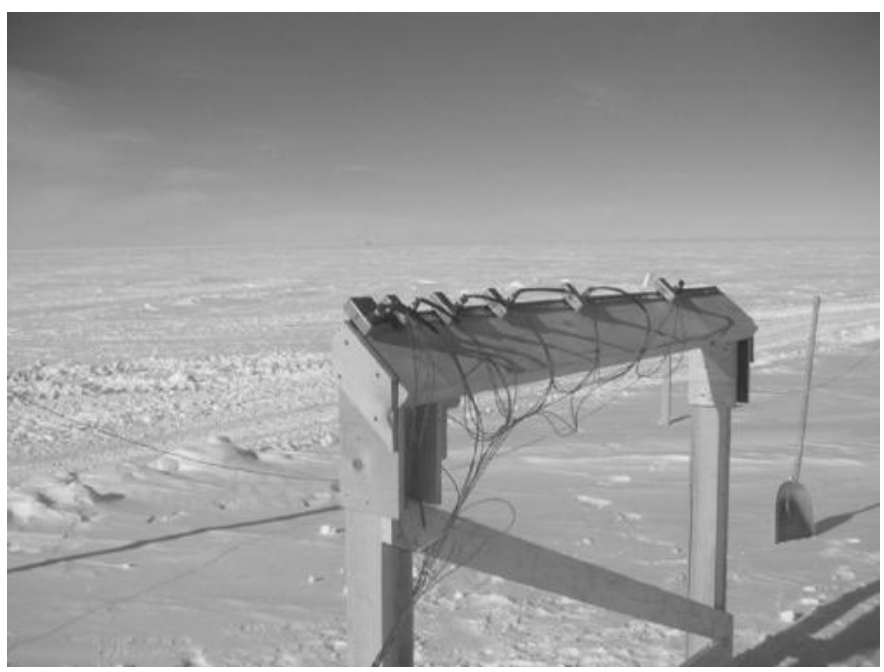

Figure 4: The 7-element lunar SHABAR orientated northwards at an elevation angle of $\sim 40 \mathrm{o}$, coinciding with the maximum lunar altitude throughout the winter season above the Dome $\mathrm{C}$ site. The SHABAR is positioned at a height of approximately 1 meter, and is located away from any source of man-made turbulence (such as the heated laboratory).

\subsection{Thermal Design}

Each SHABAR unit is sealed with a thin glass window on the front. This is to prevent ice build-up on the photodiode and/or associated electronics. A small resistor on a separate de line is included inside each unit and is switched on for a set amount of time before intensity data is recorded. The aim of this procedure is to remove any ice build-up on the outside or inside of the window.

One of the 7 SHABAR units was low temperature tested at an ambient temperature of $-80 \mathrm{C}$ and found to perform adequately. This is described below. 


\subsection{Cabling}

Much attention was paid to the cabling of the SHABAR as (1) the system noise must be as low as possible to detect the level of variation expected and (2) static build-up at Antarctic sites is known to be high. Teflon coated low temperature rated cable was used throughout. The data lines from each SHABAR unit are completely separate- each SHABAR unit has a data and return line, each a shielded twisted pair. This prevents cross-talk between the $15 \mathrm{~m}$ distance between the SHABAR location and control pc.

\section{AUTOMATED CONTROL}

The SHABAR is controlled by an industrial pc-104 system based on the supervisor system used by UNSW for the control of the AASTINO and corresponding instruments [15]. The $300 \mathrm{MHz}$ low power consumption pc saves critical data to $8 \mathrm{~GB}$ solid state flashdisk space that also contains the Linux operating system. Two 200GB standard rotary hard disk drives provide additional high volume disk space. All data is backed up to the Concordia station once per week. The $\mathrm{pc}$ is located inside the atmospheric laboratory where a temperature above $0^{\circ} \mathrm{C}$ is maintained throughout the winter period.

Power to the SHABAR units is constantly maintained. Data is recorded in bursts of 1 second duration, every 5 minute intervals at a sampling frequency of $1 \mathrm{kHz}$. The gap between reading successive photodiode signals is for all intents and purposes zero.

\section{DATA MANAGEMENT}

Data storage, transfer and communications are largely handled by the UNSW team in Sydney. A NSF supplied Iridium link enables direct connection to the supervisor computer. On a day to day basis the process of retrieving system status updates and up to $1 \mathrm{MB}$ of data per connection is automated, however, it is possible to change any feature of the system throughout the winter season. A LAN connection between the supervisor computer and Concordia station means that an automated back-up occurs once per week.

\section{THERMAL TESTING}

The SHABAR units were thermally cycled for a substantial period of time using the large biological freezer in the UNSW labs. In order to test the performance without the necessity of removing the freezer lid, a fiber optic bundle was attached to the front of a single unit, as shown in Figure 6. The bundle transferred light from a flashing LED calibration unit, located outside the low temperature environment as shown in Figure 5, to the photodiode under test. The frequency of the flashing LED could be varied from a few $\mathrm{Hz}$ to several $\mathrm{kHz}$. All SHABAR units were tested with this calibration unit to check performance. 


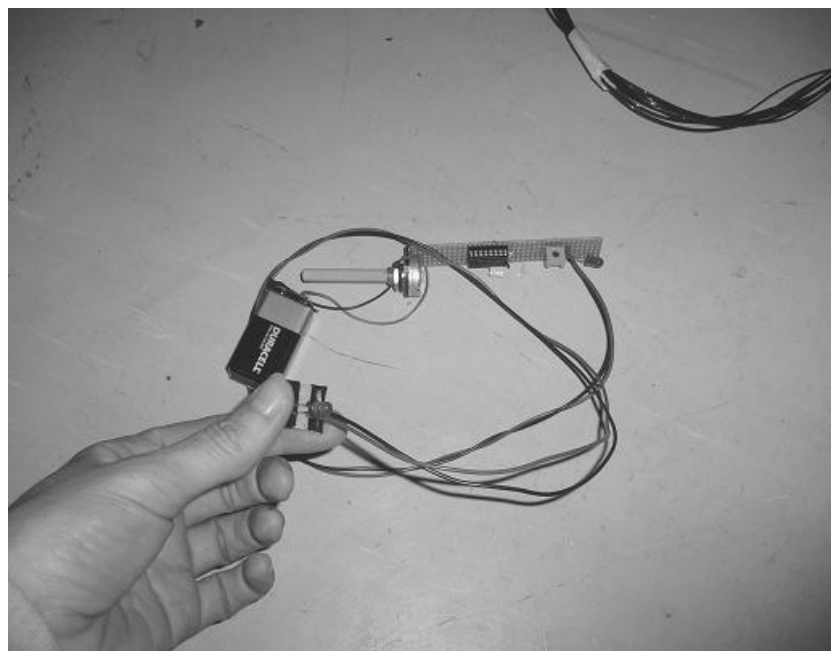

Figure 5: A simple flashing LED circuit was used to test the response of the SHABAR units

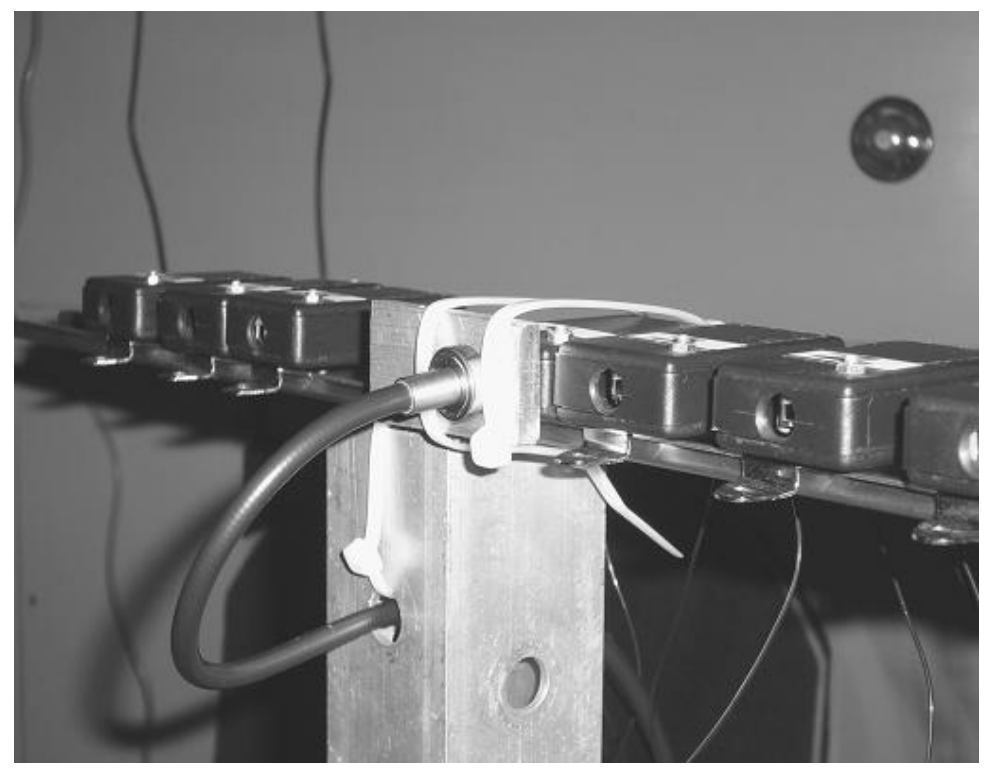

Figure 6: The SHABAR units prior to low temperature testing. A fiber optic bundle transports light from the calibration LED located outside the freezer to a single SHABAR unit, eventually located inside the freezer, at an ambient temperature of $-80^{\circ} \mathrm{C}$. 


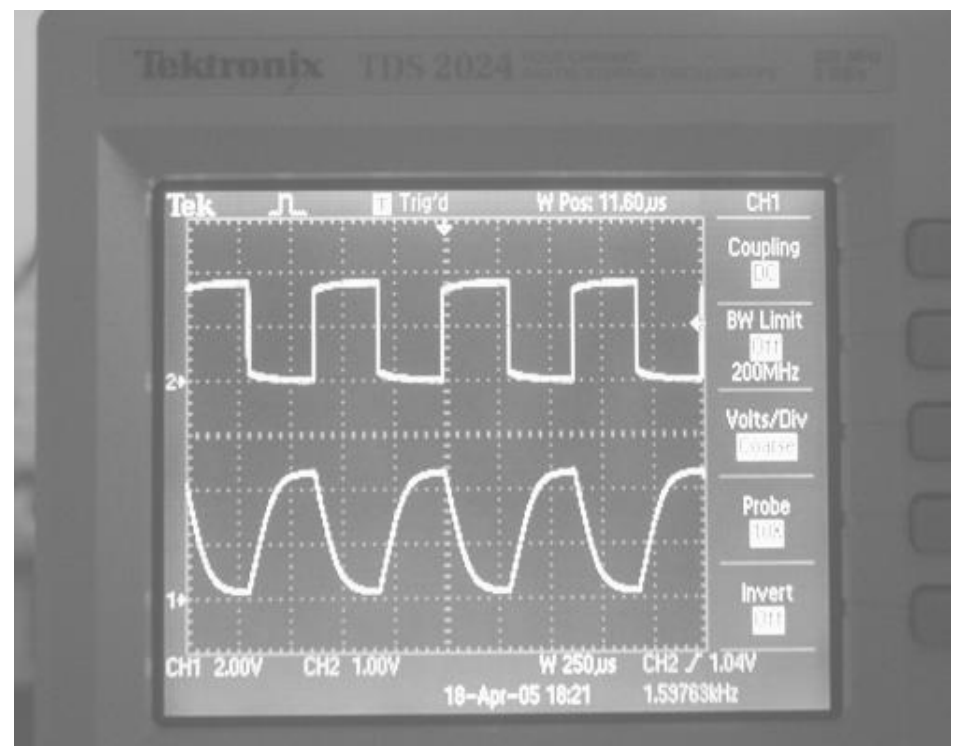

Figure 7: LED input voltage (top) and SHABAR unit output voltage as measured by the analogue to digital converter (bottom). The SHABAR unit electronics only amplify intensity frequency fluctuations that are greater than $\sim 3 \mathrm{~Hz}$.

\section{SITE TESTING}

After assembly in the UNSW labs, the SHABAR was shipped to Siding Springs Observatory and operated for a period of $\sim 1$ month. The site test provided a very useful test for software and automated control in particular.

\section{DATA}

The Dome C lunar SHABAR has been operating since installation in January 2006. Useful data awaited the setting of the sun and correct moon elevation and azimuthal position (from mid-March onwards). Except for short term power cuts at the site the data has been recorded continuously throughout the season. Samples recorded every 30 minutes are downloaded to UNSW in Sydney via the Iridium network, such as that shown in Figure 8. The full data set is saved locally at the site.

Calibration of the Dome C SHABAR is on-going, in particular correct conversion of the correlation values such as those shown in Figure 8 to reliable structure constant profiles. 

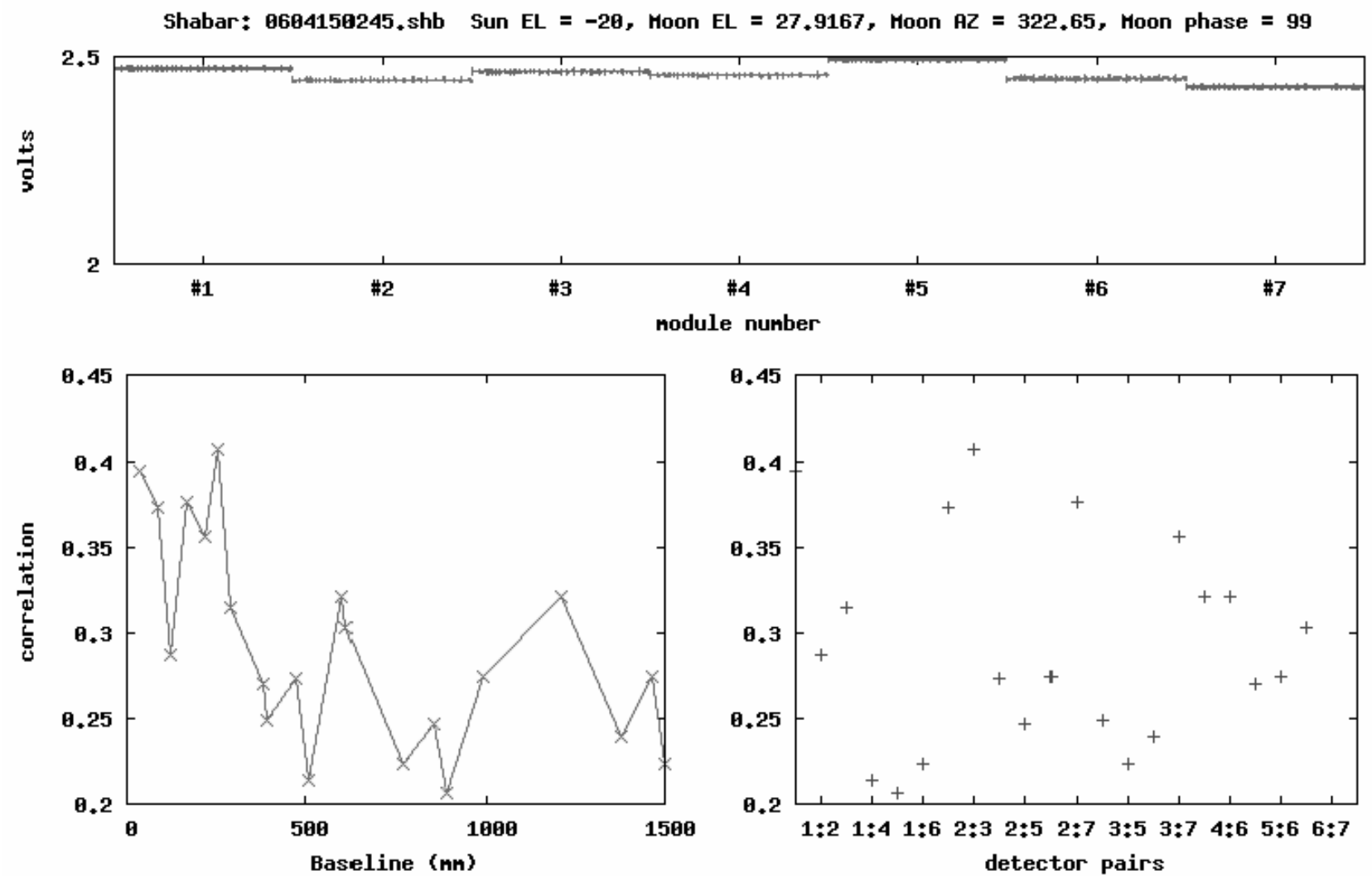

Figure 8: Sample data from the Dome C lunar SHABAR downloaded via the Iridium network to Sydney. The raw voltages from each of the 7 SHABAR units sampled by the analogue to digital card are shown (top); correlation is shown as a function of SHABAR baseline (bottom left) and as a function of the unit pairs (bottom right).

\section{CONCLUSIONS}

We present an automated transit lunar SHABAR is currently investigating the ground layer turbulence properties of the high Antarctic plateau site of Dome C. We hope for the continued operation at Dome C for further 2-3 years or more.

\section{ACKNOWLEDGMENTS}

The authors are greatly indebted to the following people and/or institutions (1) PNRA (Programma Nazionale di Ricerche in Antartide) for amazing logistical support at a timely moment (2) IPEV (Institut Polaire Francais Paul Emile Victor) and PNRA staff assistance at the Concordia station (3) the National Science Foundation for loan of an Iridium card (4) the Australian Antarctic Division and (5) Runa Briguglio, PHD student, University of Roma La Sapienza, for photographic aid at the site prior to shipment.

\section{REFERENCES}

1. Beckers, J. M. 1993, Sol. Phys., 145, 399

2. Seykora, E. J. 1991, Sol. Phys., 145, 389

3. Hickson, P., referred to in Pan-STARRS Internal Memo

4. Kaiser, N. 2004, Pan-STARRS Internal Memo

5. Aristidi et al., 2005, A\&A 444..651A

6. Lawrence, J.S., Ashley, M.C.B., Tokovinin, A., Travouillon, T. 2004, Nature, 431, 278

7. Ragazzoni, R., et al. 2005, EAS Publications Series, 14, 161

8. Lawrence, J.S., Burton, M.G., Ashley, M.C.B., \& Storey, J.W.V. 2005, EAS Publications Series, 14, 321 
9. Lloyd, J.P., Swain, M., van Belle, G.T., Coude du Foresto, V., Walker, C.K., Traub, W.A., \& Storey, J.W.V.12004, American Astronomical Society Meeting Abstracts, 205, \#181.03

10. Saunders, W., and McGrath, A.J. 2004, SPIE, 5489, 462

11. Storey, J. W. V. et al. 2006, SPIE, in press

12. Travouillon, T. 2005, EAS Publications Series, 14, 31

13. Agabi, A., Aristidi, E., Azouit, M., Fossat, E., Martin, F., Sadibekova, T., Vernin, J., Ziad, A. 2006, PASP, 118,344

14. Kenyon, S.L., \& Storey, J.W.V.2006, PASP, 118, 489

15. Lawrence, J.S., et al.2003, ESA SP-539: Earths: DARWIN/TPF and the Search for Extrasolar Terrestrial Planets, 497 